\title{
Determination with Deep Learning and One Layer Neural Network for Image Processing in MultiSlice CT Angiogram
}

\author{
Sarvadeva BhatlaMurali Krishna*, Dr. M Chandra Shekar** \\ *(PhD Scholar in ECE, at Rayalaseema University with Regd No. PP-ECE-0044, AP, India) \\ ** (Senior Manager Bharath Dynamics Limited, Hyderabad, Telangana, India)
}

\begin{abstract}
Today's world Coronary artery disease is the most common cause of death worldwide and thus early diagnosis. Well-timed opportune of this disease can lead to significant reduction in its morbidityand mortality in both younger and older for angiogram test. In this research multi slice CT scanner is used for heart angiogram test. With the help of this multi slice CT angiogram image we detect the hart diseased or not. For this disease identification and classification of angiogram images many machine learning algorithms are previously proposed those are SVM RBF and RBF neural network. Problem with SVM isnon-liner method when use any type of application will miss most liner ways of blood vessels and lack of speed in process. For non linear classification we are using RBF SVM. Problem with RBF neural network is not solve the hierarchal and component based problems, so resolve the problem using deep learning. This issue drastically improves the estimation efficiency for real time application. This methodology consumes less time for both learning as well as testing comparatively than any other methods. This issue highly improves the estimation efficiency and accuracy for real time 256, 512 slices CT scan angiogram image.
\end{abstract}

I.

\section{NTRODUCTION}

Most of the human beings are suffering Heart diseases. Some cases identification of the hart dices in particular blood vessel is difficult so, in these cases doctors suggested the angiogram test with the help of multi slice CT scan. A CT scanner is the same way as a conventional $\mathrm{X}$-ray but instead of taking one image a CT scanner takes multiple images, or slices. A computer program gathers all the images and compiles them to create a three dimensional image of the internal structures being examined. Advances have improved the sensitivity and usefulness of CT scanners since they were developed. It can provide a 3 dimensional image of an internal structure, it can detect differences in tissue density, and the $\mathrm{x}$-rays can be focused directly on specific areas, producing a clear image [1] [2].In this research we are using advanced CT scanners that are 256 and 512. Basic CTA scan 3D image is shown below Figure 1.The basic heart images of patient with coexistence of transvenous (active) and epicedial (not active) leads; A, B. Three-dimensional images of the heart; * visible patch electrodes; C1, C2. Diagnostic visualization of the coronaries: left anterior descending artery (LDA), right coronary artery despite (RCA) the presence of both types of lead; D. Multi planar reformatted reconstruction with visible artefacts from the leads[3].
I

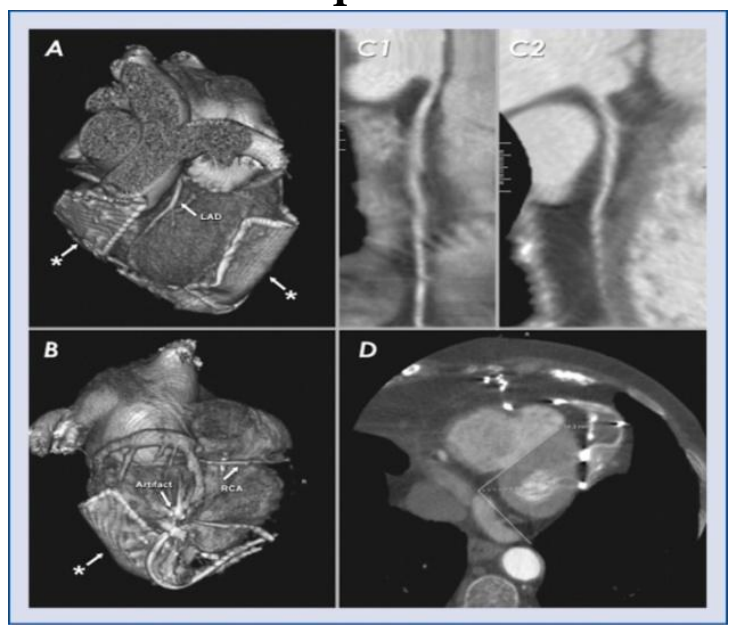

Figure1: Basic CTA Scan 3D Image

In process of medical analysis CT images used normally first step is segmentation. The research for medical image analysis accurate angiogram segmentation is a crucial prerequisite for computer-aided hepatic disease diagnosis and treatment planning. In this research Multi slice CT angiogram segmentation is performed with the help of k-means++ clustering algorithm [4].Figure 2 represents the Coronary artery disease multi slice CT scanner image with segmentation. A Curved multi-planar reformations (CMPR) of the right coronary artery (RCA), Maximal inspiratorypressure (MIP), and perpendicular cut- 
planes (Inlays a-d) delineate multiple non-calcified plaques in mid-left anterior descending (LAD), one leading to a haemodynamically significant stenos is (asterisk, cut-plane b). In the RCA, CMPR B, Virtual reality (VR) image, and MIP demonstrate multiple non-significant wall irregularities in the mid-segment (arrows) and occlusion of the distal segment (arrowheads) [5]. As shown in the internal carotid artery (ICA) $\mathbf{C}$ and $\mathbf{D}$, all lesions were correctly identified.

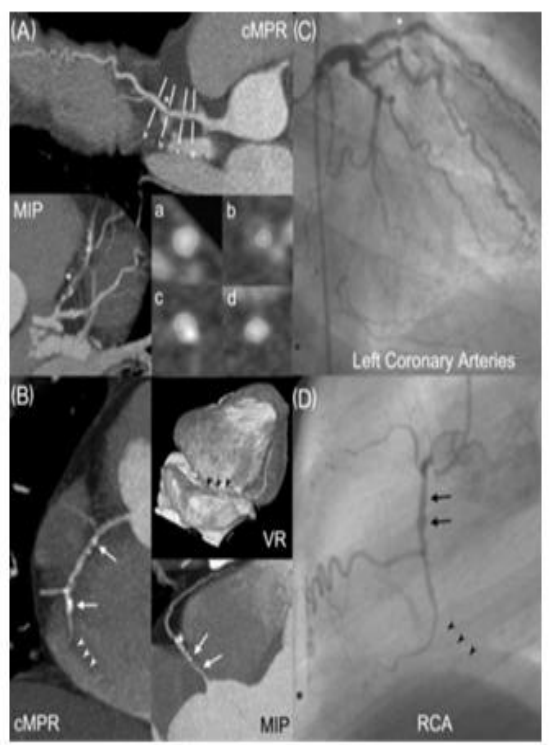

Figure 2: segmentation of CT angiogram heart image

This heart angiogram multi slice CT image Classification and retrieval is the more difficult job from fast decade, because of the exponential growth of images. The accuracy assessment of images correspondence using deep learning all are emphasized in this research [6]. All aspects of images features are covered. A total of 11 features are defined in four categories: spatial pixel level, Texture, shape and relational. In this research, all aspects of features are extracted and analyzed on different Data sets.

After extracting the features then identifies the angiogram heart image is diseased or not. For that olden days use the machine learning algorithms that are support vector machine and RBF neural network. Here SVM is Non-Liner method when use any type of application will miss most liner ways of blood vessels and lack of speed in process. For Non-Linear classification we are using RBF SVM. A common disadvantage of nonparametric techniques such as SVMs is the lack of transparency of results. RBF neural network is not solving the hierarchal and component based problems, in order to overcome this problem by using Deep Learning. This issue extremely improves the estimation efficiency of real time application. Final analysis of the research gives more improve of the accuracy [7] [8].

\section{BACK GROUND}

Nowadays, cardio- and cerebro-vascular diseases have greatly threatened human health. Established invasive test for non invasive coronary artery evaluation with high sensitivity.Cardiac automatic disease is identified with the help of angiogram test by using multislice CT scanner.

These can produce an image in less than a second and thus can obtain images of the heart and its blood vessels (coronary vessels). The First Multislice CT invention by Kalender in the 1980s, helical scan CT machines has steadily increased the number of rows of slices they deploy [9] [10]. In this research we are using advanced CT scanners that are 256-slice and 512-slice.

The 256 CT scanners have craniocaudal coverage of approximately 12 and $16 \mathrm{~cm}$, respectively. This potentially allows the heart to be scanned in one tube rotation and one heartbeat, without table movement. 512-slice CT scan has resolutions of $512 \times 512$ pixels With varying pixel sizes and slice thickness between $1.5-2.5 \mathrm{~mm}$, acquired on Philips and Siemens Multi-Detector CT scanners with $120 \mathrm{kVp}$ tube voltage [11]. This heart angiogram Multislice CT Image Classification and retrieval is the more difficult job from fast decade, because of the exponential growth of images [12]. The accuracy assessment of images correspondence using Deep Learning all are emphasized in this research.

\section{PROPOSED METHODOLOGY}

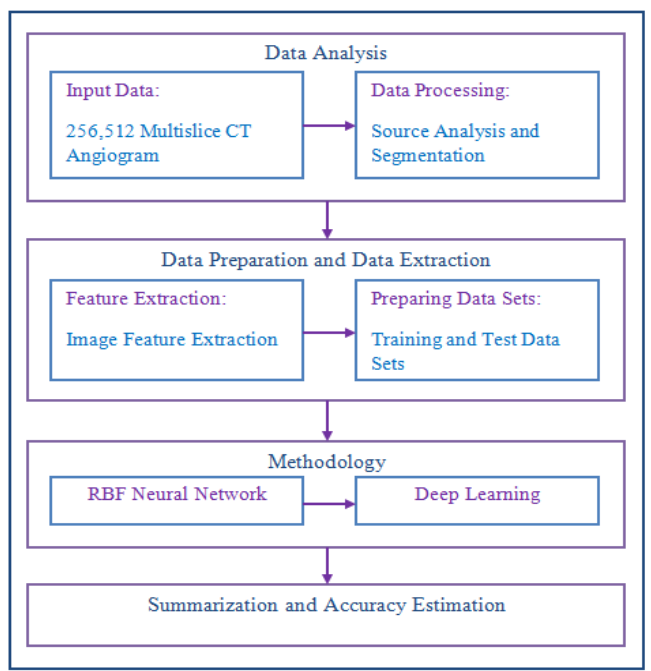

Figure 3: Flow chart of proposed methodology architecture

Major process divided into four steps: 
- The initial step is data preparation;Data analysisin this first collecting the data from data sets after that this data is pre-processed before data selection.

- Subsequently data processing the entire data is grouped into different datasets. Each data set consists of Train Data sets and Test Data sets. Test and Train data set whose details are explained in the next sections.

- Next followed step is segmentation to extract Feature from trained and testdata sets.

- The heart Step for this research is Deep Learning system.

- After selecting the method build the system first and then test with different test cases for evaluating the performance in terms of accuracy.

\section{TEST DATA}

In this Research, data werecollected from Agecanonix, FIVIX and MAGIX are the most widely used data sets for many research problems. This datasets consisting of different kinds of Multislice CT Heart.Angiogram images. The images are of the size 128X128, 256X256 and 512X512. This research used the Agecanonix, FIVIX and MAGIX image databases, which is consisting of 1106, 1754, 760 Images. Each data set consisting of minimum three subjects.Each subject consisting of 50 to 100 angiogram images.

Agecanonix data set is contains 13 subjects. First subject consisting of 6 records and 355 images. From second subject to ninth subject contain equal amount of images, but different no. of records. 10 and 11 subjects contain different no. of records, but equal no. of images. The entire Agecanonix data set is represented in below bar chart figure 4.

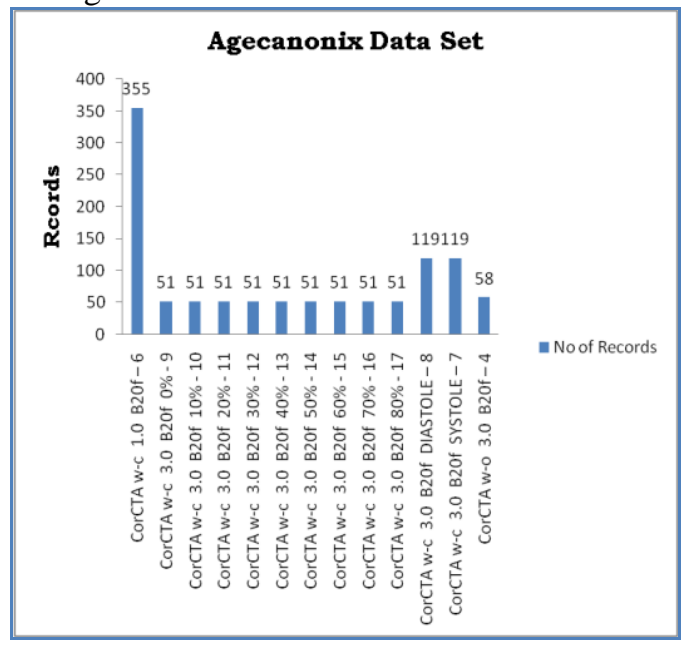

Figure 4: Agecanonix Data Set Bar C Hart

FIVIX data set is contains 3 subjects. First subject contain 11 records and 414 images. Second subject contains 13 records and 530 images. Final subject contain more no. Of records 404 and more images 810 . Entire 3 subject's relation is shown in below figure 5 .

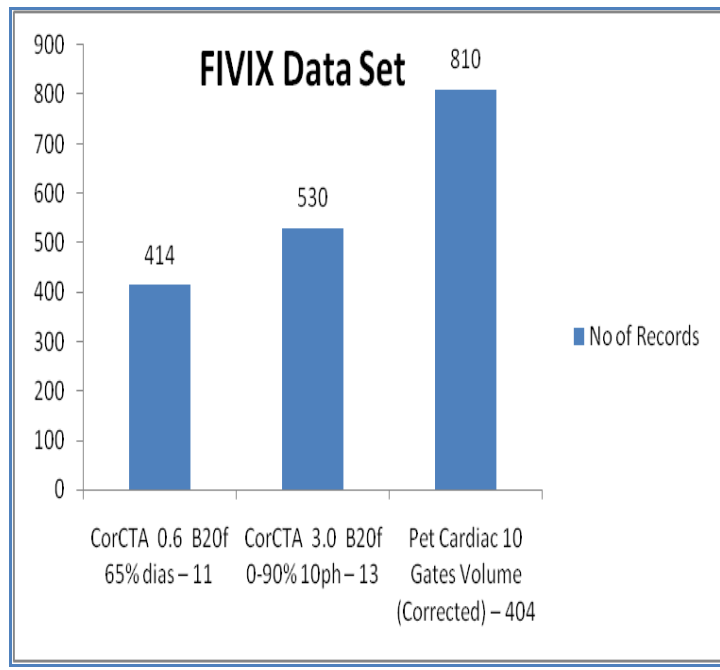

Figure 5: FIVIX Data Set Bar Chart

MAGIX data set is contains 10 subjects. All the subjects' records are different, but it contains equal no. of images. It shown in below bar chart 6.

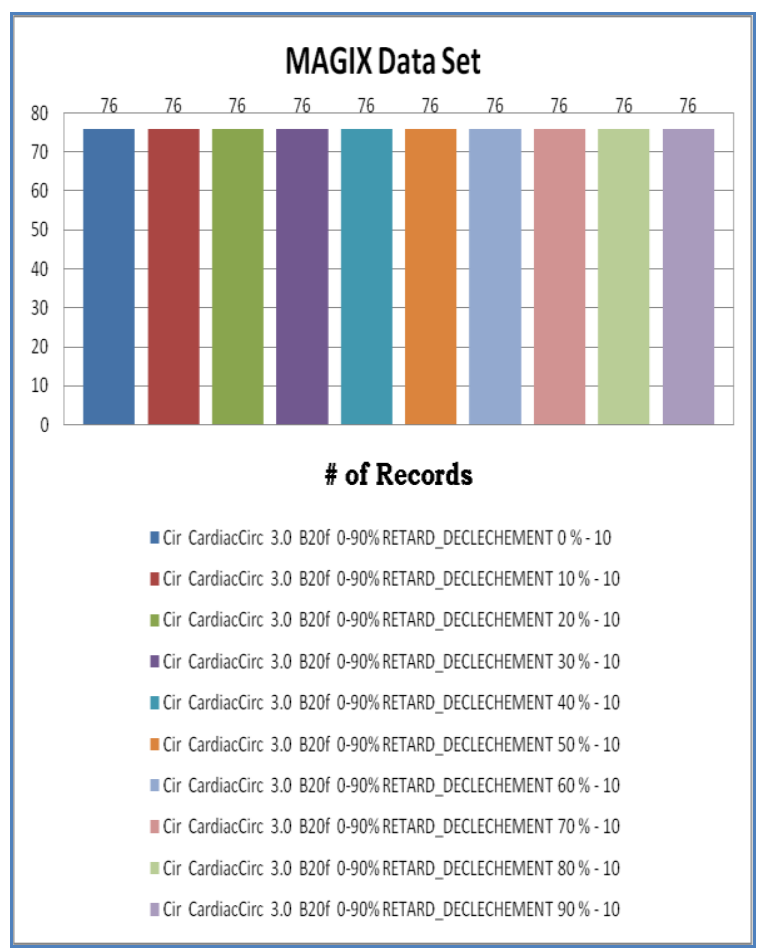

Figure 6: MAGIX Data Set Bar Chart

In this research 3 data sets are using .each data set is containing multiple no of angiogram heart images. After that extract the feature sets from these data sets. 


\begin{tabular}{|l|l|}
\hline Technique Name & Feature Name \\
\hline Statistical Pixel-Level & Mean \\
\hline Statistical Pixel-Level & Variance \\
\hline Statistical Pixel-Level & Gray Value \\
\hline Statistical Pixel-Level & Contrast of Pixel \\
\hline Statistical Pixel-Level & Edge Gradient \\
\hline Shape & Circularity \\
\hline Shape & Compactness \\
\hline Shape & Moments \\
\hline Shape & Chain Codes \\
\hline Relational & Relational Structure \\
\hline Relational & Hierarchical Structure \\
\hline
\end{tabular}

Table 1: Feature Extraction Descriptions

\section{RBF NEURAL NETWORKS}

The Radial Basis Function Neural Network (RBF NN) is a three layer design with weights and bias optimization values.Generally in Radial Basis Function, input layer consists of input data which is extracted from image features. This work used different dimensions of the image features like statistical pixel level, Texture, shape and Relational.In the hidden layer, it contains one additional node than input node. This layer consists of Centroids which are calculated by using Gaussian distribution function internally. Outputs are computed from processing layer and hidden layer plus Bias values.It is represented in below equation 1(13).

Output Layer $=$ Sum of (Hidden Layer $*$ Weights $)+$ Bias value.

Figure 7, shows the block diagram of RBF Neural network. The input, output and hidden layers are $\mathrm{J} 1, \mathrm{~J} 2$, J3 neurons respectively.

$\phi\left(\mathrm{x}^{-}\right)=1$

Above equation 2 represents the bias in the output layer below equation 3 represents the non linearity at hidden nodes

$\phi\left(\mathrm{x}^{-}\right)$

\section{(3)}

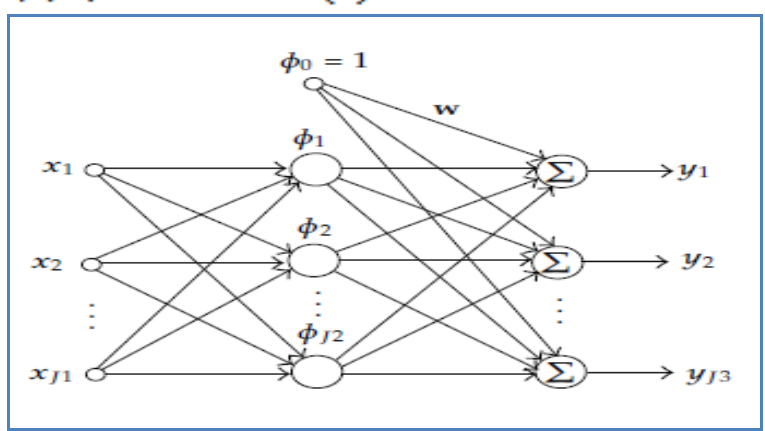

Figure 7: Block diagram of RBF neural network
Where $y_{i}\left(x^{-}\right)$is the $i_{\text {th }}$ output, $w_{k i}$ is the connection weight from the $\mathrm{k}_{\mathrm{th}}$ hidden unit to the $\mathrm{i}_{\text {th }}$

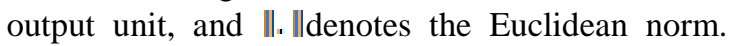
The RBF $\phi(x)$ is typically selected as theGaussian function, and such an RBF network is usually termed the Gaussian RBF network. The output function $f$ compute by using the below approximation function like below equation 4 (14).

$$
y_{i}(\vec{x})=\sum_{k=1}^{J_{2}} w_{k i} \phi\left(\left\|\vec{x}-\vec{c}_{k}\right\|\right), \quad i=1, \ldots, J_{3},
$$

In this research Neural Network and Deep Learning are proposed for the learning and classification of Multi Slice CT Angiogram images. But RBF neural network is not solving the hierarchal and component based problems, in order to overcome this problem by using Deep Learning. This issue extremely improves the estimation efficiency of real time application (15).

\section{DEEP LEARNING}

In Neural networks good weights learning algorithm is RBF NN with1 hidden layer, explained in. Not good at learning the weights for networks with more hidden layers. The problem may not solve one layer, but our research requiredfor Multi types of futures to resolve the problem. Because blood vessels is not signal types of features extraction (16).

A Deep Neural Network is an Artificial Neural Network with multiple hidden layers of units between the input and output layers. DNN is used in computer vision. Deep Learning algorithms for Unsupervised or Semi Supervised feature learning and hierarchical feature extraction algorithm.

The first Supervised Deep Learning was invented by Ivakhnenko and Lapa in 1965. Artificial Neural Networks was introduced by Igor Aizenberg and colleagues in 2000. Unsupervised representation learning methods such as Restricted Boltzmann Machines (RBM) may outperform standard filter banks because they learn a feature description directly from the training data(17). 


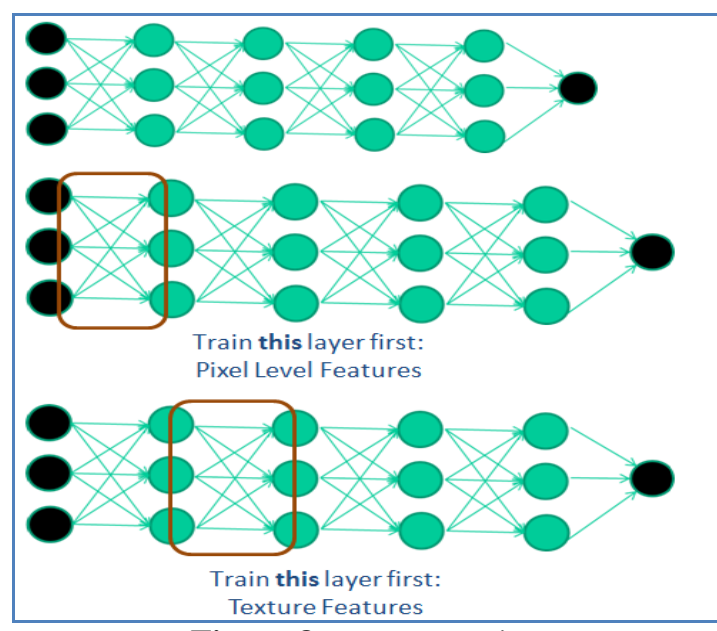

Figure 8: Deep Learning

Like this above figure 7 each layer our research different future data sets with layer output

\section{ACCURACY ASSESSMENTS}

In this paper of research are used three different data sets. About data sets are specified in section 4.Each data set, divided into some set of data has training data set and left our part is test data set. Each data set contained the heart angiogram images this data is used to identify the diseased blood vessels in the human heart. Instance of data set is already defined how much blood vessels part of data sets for train data. Has first training data set is defined $90 \%$ data set from original data and $10 \%$ consider has test data set. Like that, decrease training data set $\%$ and increase test data set $\%$ with different 7 cases are

\begin{tabular}{|c|c|c|}
\hline $\begin{array}{c}\text { Seq. No } \\
\text { Case }\end{array}$ & $\begin{array}{c}\text { Training Data } \\
\text { Set \% from } \\
\text { Original Data } \\
\text { Set }\end{array}$ & $\begin{array}{c}\text { Test Data } \\
\text { Set \% from } \\
\text { Original } \\
\text { Data Set }\end{array}$ \\
\hline Case \#1 & 90 & 10 \\
\hline Case \#2 & 80 & 20 \\
\hline Case \#3 & 70 & 30 \\
\hline Case \#4 & 60 & 40 \\
\hline Case \#5 & 50 & 50 \\
\hline Case \#6 & 40 & 60 \\
\hline Case \#7 & 30 & 70 \\
\hline
\end{tabular}

Table 2: Training and Test Data Sets \% with Different Test Cases

Used with above training and test data sets for each analysis division of $\%$ shown in above Table 2. Assessment of accuracy done for following methods with above train and test data set cases. Those are shown below table 3
- $\quad$ RBF neural network

- Deep Learning.

\begin{tabular}{|c|c|}
\hline Cases & Accuracy of RBF NN \\
\hline Case \#1 & $96 \%$ \\
\hline Case \#2 & $95 \%$ \\
\hline Case \#3 & $95 \%$ \\
\hline Case \#4 & $95 \%$ \\
\hline Case \#5 & $90 \%$ \\
\hline Case \#6 & $86 \%$ \\
\hline Case \#7 & $85 \%$ \\
\hline
\end{tabular}

Table 3: Accuracy of RBF NN

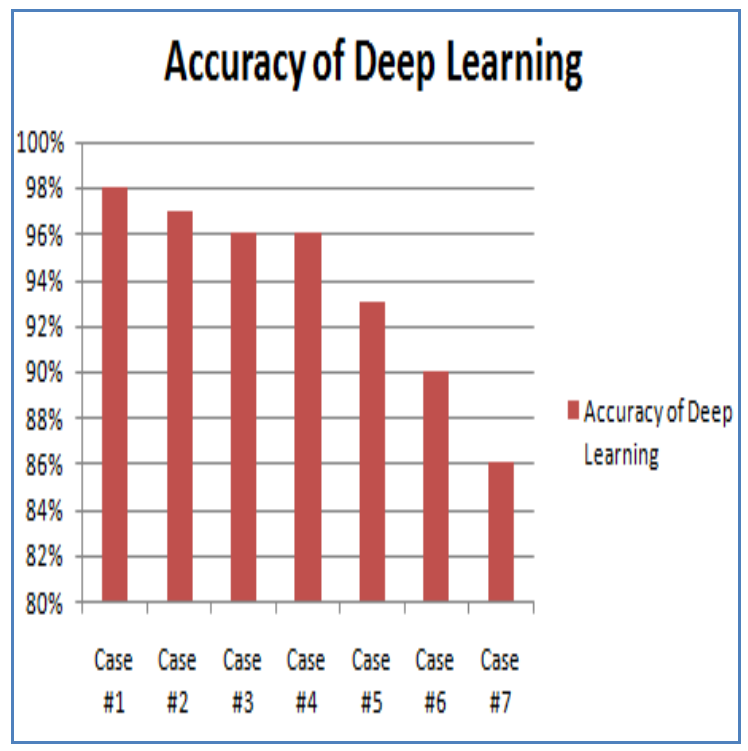

Figure 9: Bar Chart for Accuracy of RBF NN

\begin{tabular}{|l|l|}
\hline Cases & $\begin{array}{l}\text { Accuracy of Deep } \\
\text { Learning }\end{array}$ \\
\hline Case \#1 & $98 \%$ \\
\hline Case \#2 & $97 \%$ \\
\hline Case \#3 & $96 \%$ \\
\hline Case \#4 & $96 \%$ \\
\hline Case \#5 & $93 \%$ \\
\hline Case \#6 & $90 \%$ \\
\hline Case \#7 & $86 \%$ \\
\hline
\end{tabular}

Table 4: Accuracy of Deep Learning 


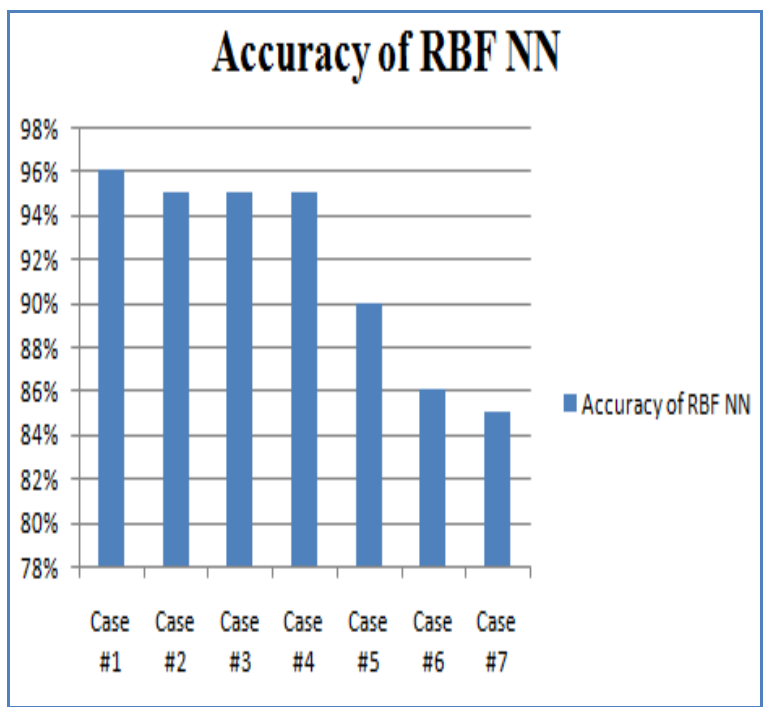

Figure 10: Accuracy of Deep Learning

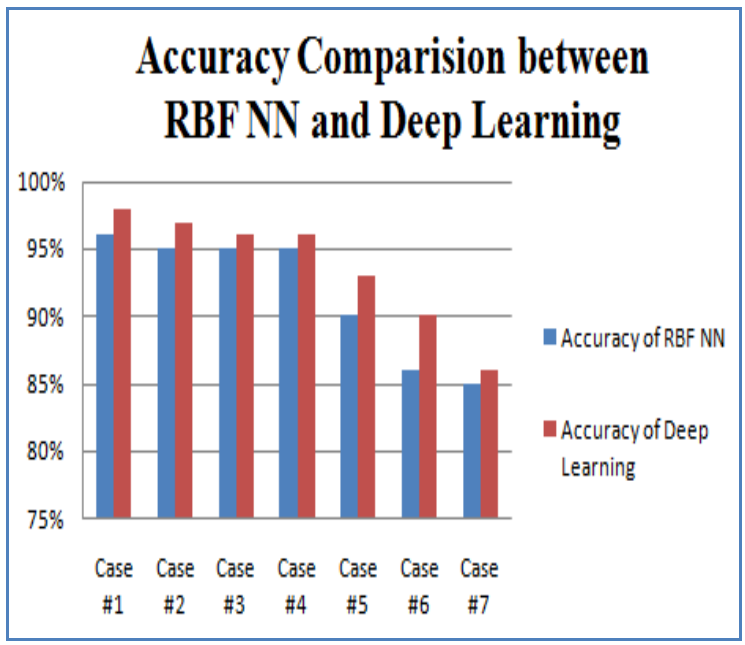

Figure 11: Bar Chart for Acc Comparison between RBF NN and Deep Learning

Accuracy is exhibited above Bar Chart for Acc Comparison BW RBF NN and Deep Learning for research methodologies. Clear proved Deep Learning is more accurate compare than RBF NN and another thing is here clear specified even decrease train data set also accuracy is stabilized in all aspect not much decrease as like RBF NN [18].

Additional analyzed RBF NN stabilized different with Weights and Bias values. Total weight values are 132 values and 2 bias values.

\section{CONCLUSIONS}

This paper, proposed a method to find the Coronary Heart diseases. In this research using Computed Tomography (CT) scanner for identified blood vessel in heart area. The problem of identification blood vessels in heart is binary classification. Majorly binary classification algorithmis SVM. SVM is the Non- linear classification, so it misses more no. of linear blood vessels. In order to overcome this problem implements the RBF NN. It is the both linear as well as Non-linear classification.RBF neural network is not solving the hierarchal and component based problems, in order to overcome this problem by using Deep Learning. Hence, this paper gives clear information about improved classification using deep learning compare with existing methodology of RBF neural network.

Future extended with advanced methodology for image classification, feature extractions and best accuracy assessment.

\section{REFERENCES}

[1]. Lei Z, Han P, Xu H, Yu J. Application study of 512-slice computed tomography low dose coronary angiography. Digit Med 2015; 1:28-33.

[2]. Sun ZH, Cao Y, Li HF. Multislice computed tomography angiography in the

[3]. diagnosis of coronary artery disease.J Geriatr Cardiol. 2011 Jun;8(2):104-13. doi:10.3724/SP.J.1263.2011.00104.

[4]. Thayssen P, Jensen LO, Lassen JF, et al. The risk and prognostic impact of definite stent thrombosis or in-stent restenosis after coronary stentimplantation.

EuroIntervention 2012;8(5):591-8.

[5]. Rixe J, Achenbach S, Ropers D, et al. Assessment of coronary artery stent restenosis by 64-slice multi-detector computed tomography. Eur Heart J 2006;27(21):2567-72.

[6]. Jensen JK, Jensen LO, Terkelsen CJ, et al. Incidence of definite stent thrombosis or instent restenosis after drug-eluting stent implantation for treatment of coronary instent restenosis. Catheter Cardiovasc Interv 2013;81(2):260-5.

[7]. Pugliese F, Cademartiri F, van Mieghem C, et al. Multidetector CT forvisualization of coronary stents. Radiographics 2006;26 (3):887-904.

[8]. Jung JI. Multidector CT imaging of coronary artery stents: is this method ready for use? Korean Circ J 2007;37(11):521-9.

[9]. Quoc, L., Ngiam, J., Chen, Z., Chia, D., Koh, P. W., and Ng, A. Tiled convolutional neural networks. In NIPS 23. 2010.

[10]. Vinyals, O., Jia, Y., Deng, L., and Darrell, T. Learning with Recursive Perceptual Representations. In NIPS, 2012.

[11]. Xu L, Sun Z. Virtual intravascular endoscopy visualization of calcified coronary plaques: a novel approach of identifying plaque features for more accurate assessment of coronary lumen stenosis. 
Medicine. 2015; 94(17):e805. doi: 10.1097/MD.0000000000000805 PMID: 25929936; PubMed Central PMCID: PMC4603061.

[12]. Almoudi M, Sun Z. Coronary artery calcium score: Re-evaluation of its predictive value for coronary artery disease. World journal ofcardiology. 2012; 4(10):284-7. doi: 10.4330/wjc.v4.i10.284 PMID: 23110244; PubMed Central PMCID: PMC3482621.

[13]. Syeda-Mahmood, T., Wang, F., Beymer, D., Amir, A., Richmond, M., Hashmi, S. Aalim. "Multimodal mining for cardiac decision support". Comput. Cardiol. 34, 209-212 (2007).

[14]. K.-L. Du and M. N. S. Swamy, Neural Networks in a Softcomputing Framework, Springer, London, UK,2006.

[15]. Hofman MBM, Paschal CB, Li D, et al. MRI of coronary arteries: 2D breath-hold vs 3D respiratory-gated acquisition. J Comput Assist Tomogr.

[16]. Saman Sarraf, and Ghassem Tofighi, "Classification of Alzheimer's Disease using fMRI Data and Deep Learning Convolution Neural Networks," arXiv preprint arXiv: 1603.08631,2016.

[17]. S. S. Watkins and P. M. Chau, "A radial basis function neuron computer implemented with analog VLSI circuits," in Proceedings of the International Joint Conference on Neural Networks, vol. 2, pp. 607-612, Baltimore, Md, USA. 